\title{
Acceleration of high charge ion beams with achromatic divergence by petawatt laser pulses
}

\author{
S. Steinke, J. H. Bin, J. Park, Q. Ji, K. Nakamura, A. J. Gonsalves, S. S. Bulanov, M. Thévenet, \\ C. Toth, J.-L. Vay, C. B. Schroeder, C. G. R. Geddes, E. Esarey, T. Schenkel, and W. P. Leemans \\ Lawrence Berkeley National Laboratory, Berkeley, California 94720, USA
}

(Received 23 July 2019; accepted 4 February 2020; published 19 February 2020)

\begin{abstract}
We present a study of laser-ion acceleration, where an increased laser spot size leads to sheath field geometries that accelerate ion beams of narrow and achromatic divergence at unprecedented charge densities, resulting from the high aspect ratio of the laser spot size to the acceleration distance. Matching the laser pulse length to the transverse laser spot size mitigated laser sweeping across the target front side and optimized the acceleration, with maximum energies deviating significantly from a linear intensity scaling.
\end{abstract}

DOI: 10.1103/PhysRevAccelBeams.23.021302

\section{INTRODUCTION}

Laser-driven acceleration of ion beams [1-3] has great potential for numerous applications such as heavy ion therapy of oncological diseases [4], injectors for conventional accelerators [5], radiography [6], studies of radiation damage [7] and single event effects in electronics [8,9], as well as fast ignition inertial confinement fusion [10-13], and drivers and probes for the studies of warm dense matter $[14,15]$. Such ion beams are accelerated in the form of neutralized bunches by means of spatially localized and temporally synchronized fields from relativistic laser plasma interactions, and exhibit exceptional beam properties [6] (e.g., low emittance, short bunch duration and high charge).

For applications including those above and detailed physics studies of the relativistic laser-plasma interaction, high repetition rates $(\mathrm{Hz}$ to $\mathrm{kHz})$ are essential. During the past few years, several petawatt laser systems with short pulse duration $(<100 \mathrm{fs})$ [16] and high repetition rate (up to $1 \mathrm{~Hz}$ [17]) have become available. Such short pulse lasers are scalable to higher repetition rates and have a much smaller footprint. Thus, the path towards multipetawatt systems with intensities in excess of $10^{22} \mathrm{~W} / \mathrm{cm}^{2}$ (required to reach e.g., $400 \mathrm{MeV} / \mathrm{u}$ carbon ion energies for deep tumor carbon therapy) becomes much more cost effective [18].

\footnotetext{
*ssteinke@lbl.gov

Present address: Deutsches Elektronen-Synchrotron DESY, 22607 Hamburg, Germany.
}

Published by the American Physical Society under the terms of the Creative Commons Attribution 4.0 International license. Further distribution of this work must maintain attribution to the author(s) and the published article's title, journal citation, and DOI.
The acceleration mechanism studied most extensivelytarget normal sheath acceleration (TNSA) [19-21] — has matured to a high level of robustness and reliability. This has made studies possible that cover a wide range of laser and target parameters from many different experiments $[2,3]$. However, not all the laser-plasma and ion parameters can be measured at the same time in a single experiment, especially at petawatt-class laser systems where shot rates have historically been at or below a few shots/hour [16]. Providing comparable experiment conditions at different facilities is hardly possible, mainly due to the unique temporal intensity structure of each laser system. To date, proton energies of up to $85 \mathrm{MeV}$ [22] with TNSA, or $94 \mathrm{MeV}$ protons [23] and $1 \mathrm{GeV}$ carbon ions [24] with advanced mechanisms were generated using petawatt lasers with pulse durations exceeding 0.5 ps at ultrarelativistic intensities of $\sim 10^{20} \mathrm{~W} / \mathrm{cm}^{2}$. Proton beam divergences in TNSA are chromatic with typical values ranging from 400 to $900 \mathrm{mrad}$ at half of the maximum energy.

We report on the generation of proton beams with achromatic, narrow divergence at unprecedented charge density by reducing the curvature of the hot election sheath field on the rear side of the target. In contrast to earlier work $[25,26]$, this is achieved by increasing the laser spot size $2 w_{0}$ incident on the targets front side to values well above the target thickness $\left(l \ll w_{0}\right)$, with $w_{0}$ being the radius at $1 / e^{2}$ of the peak intensity. As the laser spot size is increased (at constant laser intensity) and hence, the virtual source size of the ion beam, the number of accelerated ions is increased accordingly. If the laser is incident at a 45 degree angle to maximize laser absorption, the laser-target interaction is prolonged by $2 w_{0} / c$, where $c$ is the speed of light, as the laser sweeps across the target. In the large spot size regime, this prolongation of the interaction is significant as it exceeds the initial laser pulse duration by a factor of 6 . 
Geometric considerations suggest that the initial laser pulse duration has to be increased to $2 w_{0} / c$ to optimize the acceleration process as well as generating a flattop-shaped electric field that leads to an achromatic divergence.

These findings are enabled by the first study of TNSA with statistical significance at petawatt laser power, consisting of several hundred shots. We applied a tape drive target system [27] that allows for conducting the experiments with titanium (Ti) tape of $5 \mu \mathrm{m}$ thickness at repetition rates up to $0.5 \mathrm{~Hz}$. Such ion beams are well suited for subsequent high gradient and emittance preserving beam transport which typically have narrow angular acceptance, such as active plasma lenses [28] and many applications in cell biology, high energy density physics and material sciences.

\section{EXPERIMENTAL RESULTS}

For these experiments, laser pulses with durations $t_{L}=$ $35-700 \mathrm{fs}$ at a central wavelength $\lambda_{L}=815 \pm 20 \mathrm{~nm}$ were generated at the BELLA PW Ti:sapphire laser [17] with a repetition rate of $1 \mathrm{~Hz}$. The laser pulses were focused using a $13.5 \mathrm{~m}$ focal length off-axis parabolic mirror to a focal spot size of $w_{0} \approx 52 \mu \mathrm{m}$. Deformable mirror (DM) based feedback technology enabled a high focal spot quality with a Strehl ratio $>0.8$. The laser energy delivered at the focal location was $E_{L}=35 \mathrm{~J}$, corresponding to peak intensities $I_{L}$ in the range of $I_{L}=0.6-12 \times 10^{18} \mathrm{~W} / \mathrm{cm}^{2}$ (depending on laser pulse duration), i.e., in terms of the normalized laser vector potentials $a_{0}=0.5-2.4$. Freestanding Ti foils of thickness $l=1-10$ micron were irradiated at a 45 degree angle of incidence in the polarization plane of the laser. The accelerated ion beams were analyzed using a Thomson parabola spectrometer in target normal direction. The characteristic parabolas were visualized by a CCD coupled to a microchannel plate (MCP) with phosphor screen [29].

The measured maximum energies for proton and carbon $\left(\mathrm{C}^{4+}\right)$ ions are plotted in Fig. 1(a). For each thickness, the focus location relative to the target surface was varied (a)

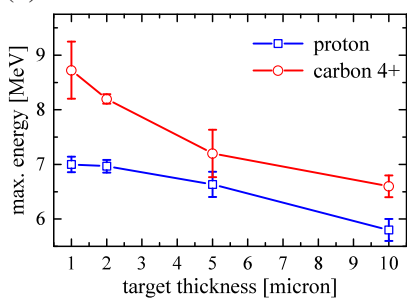

(b)

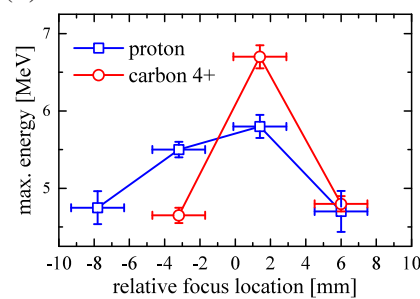

FIG. 1. Measured maximum ion energies for proton (blue) and carbon $\left(\mathrm{C}^{4+}\right)$ ions (red) in $\mathrm{MeV}$ as a function of Ti-target thickness (a) and relative focus-target distance (b) adjusted with the deformable mirror for the $10 \mu \mathrm{m}$ target. The vertical error bars represent the standard deviation from shot-to-shot variation over five consecutive shots [(a) and (b)]. The horizontal error bars represent the accuracy of the focus positioning using the DM (b). within a Rayleigh length of the laser using the DM as shown in Fig. 1(b) exemplary for the 10 micron target. The highest ion energies were obtained with the focal plane close to the target surface, i.e., with the highest laser intensity. An additional five shots were taken at the optimum focus location for each thickness for the data in Fig. 1(a). The highest energies for protons and $\mathrm{C}^{4+}$ ions were approximately 7 and $8.5 \mathrm{MeV}$, respectively.

A feedback-controlled tape drive system [27,30,31] spooling Ti tape of 5 micron thickness (and $100 \mathrm{~mm}$ width) was employed to increase the number of shots, enabling ion beam generation near the repetition rate of the laser. With this tape drive, uninterrupted operation at up to $0.5 \mathrm{~Hz}$ was possible for hours of run-time, but was limited to several hundred shots due to maximum length of the Ti tape $(5 \mathrm{~m})$. The tape position was referenced by polished metallic rods and it was tensioned before each shot to provide a wrinkle-free surface with a surface position repeatability $<10$ micron [27]. In Fig. 2(a) proton spectra

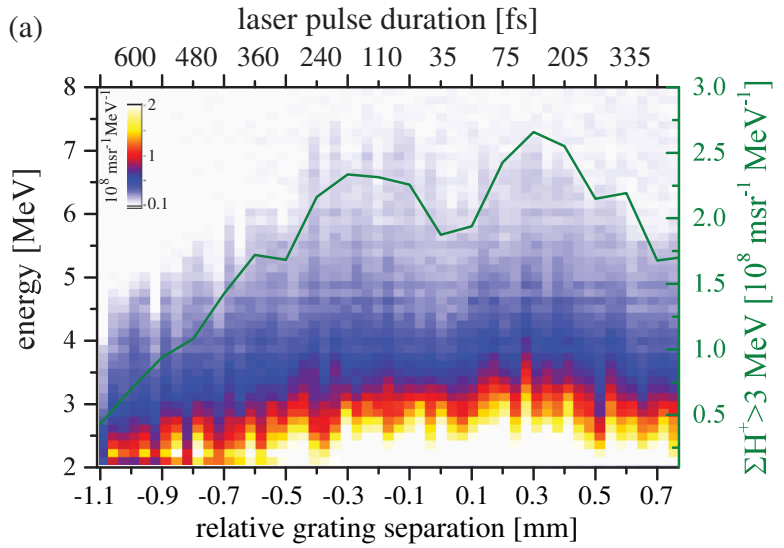

(b)

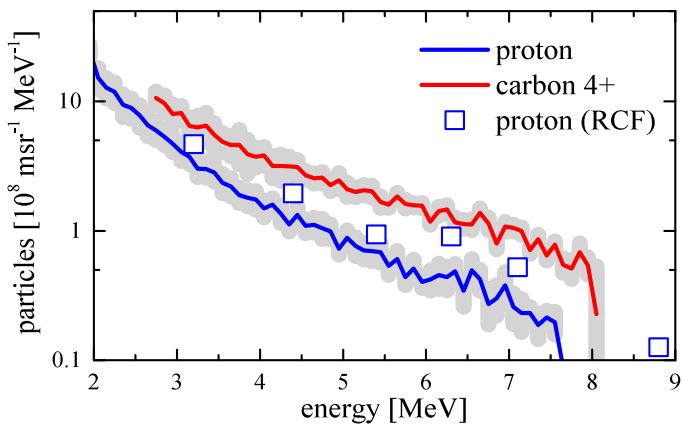

FIG. 2. Results from tape drive: (a) Waterfall plot of proton spectra as a function of relative compressor separation, i.e., laser pulse duration (positive grating separation corresponds to negative chirp and vice versa). The green line gives the integrated number of protons with energies above $3 \mathrm{MeV}$. (b) Proton (blue line), $\mathrm{C}^{4+}$ (red line) spectra averaged over 15 shots for a pulse duration of 120 fs and proton counts from radiochromic film (RCF) on the spectrometer axis (blue squares). The standard deviation from shot-to-shot variations is shown in grey for each spectrum. 

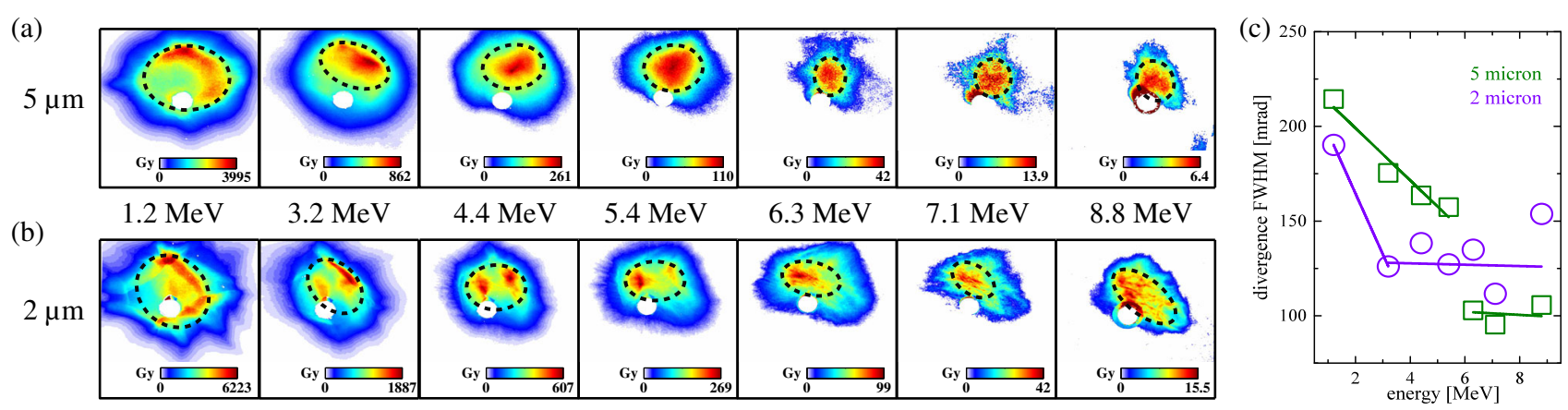

FIG. 3. Processed radiochromic film data: proton beam profiles originating from 5 micron (a) and 2 micron (b) thick Ti targets. The energy of the fully stopped protons is given in $\mathrm{MeV}$ for each film layer. The deposited dose (Gy) in each layer is given by the color scale of the individual image. The divergence was derived as the FWHM of a fitted Gaussian profile to the measured dose distribution and shown for 5 micron (green squares) and 2 micron (purple circles) for each layer, i.e., proton energy in (c). The solid lines are piecewise linear fits of the data. The visible holes in the RCF film do not correspond to the target normal direction.

are shown as a function of laser pulse duration, ranging from 35 to $660 \mathrm{fs}$ obtained with the Ti tape drive target system. The laser pulse duration was varied by changing the relative grating separation in the final vacuum compressor and thereby adding positive or negative chirp to the laser pulse. The maximum proton energy increased linearly with decreasing laser pulse duration, starting with $4.5 \mathrm{MeV}$ at $660 \mathrm{fs}$ and reaching an optimum of $7.5 \mathrm{MeV}$ at around $180 \mathrm{fs}$. For shorter pulse durations, the energy decreased to $6 \mathrm{MeV}$ at $35 \mathrm{fs}$. This behavior was independent of the laser chirp direction within our experimental accuracy.

The deviation from a linear intensity scaling [32-34] for laser pulses shorter than $2 w_{0} / c$ can be illustrated by considering the following three cases at a 45 degree angle of incidence: (i) $t_{L} \ll 2 w_{0} / c=204 \mathrm{fs}$ (FWHM): The laser interacts with the target and heats the plasma very localized $\left(\sqrt{2} t_{L} c\right)$ as it sweeps across the focal plane. At the time when the spatiotemporal intensity peak arrives at the target, the interaction is already terminated at the leading edge. The interaction time at a single point on the surface is insufficient to create plasma profile that allows for efficient coupling. (ii) $t_{L}=2 w_{0} / c$ : After the initial sweep across the focal plane, the target is illuminated and heated uniformly. (iii) $t_{L} \gg 2 w_{0} / c$ : The scenario is similar to (ii) but at reduced laser intensity and hence, reduced electron temperature $T_{e}$ according to $T_{e} \propto a_{0}$ (see Fig. 2).

Two-dimensional energy-resolved proton beam profiles were measured for target thicknesses of 5 and 2 micron [Figs. 3(a) and 3(b)] by means of radiochromic film (RCF) stacks at a laser pulse duration of $100 \mathrm{fs}$. Those RCF stacks $(5 \times 5) \mathrm{cm}^{2}$ were positioned normally $10 \mathrm{~cm}$ downstream of the target rear surface and consisted of eight layers GafChromic film, allowing sufficient sensitivity and resolution for the whole detectable energy range of the proton beams. A 15 micron thick aluminum foil was placed in front of the RCF stacks to block laser light as well as heavy ions. Each of the individual films of the layer records the deposited energy and the whole stack constitutes an energy resolved measurement. The dose response of the films and scanner unit was calibrated at the NDCX-II [35] facility with proton beams at $1.1 \mathrm{MeV}$. Reconstructed proton beam profiles are shown in Fig. 3 for shots on targets with 2 and 5 micron thickness up to the seventh layer of RCF, corresponding to proton energies of $8.8 \mathrm{MeV}$. Similar to [36], the spatially resolved proton spectra were reconstructed from the single shot dose distribution and is in agreement with our Thomson spectrometer data [see Fig. 2(b)]. In case of the 5 micron targets, the divergence decreases linearly with energy up to $5.4 \mathrm{MeV}$, followed by a constant divergence of $100 \mathrm{mrad}$ for energies ranging from 6.3 to $8.8 \mathrm{MeV}$. Such an achromatic divergence was observed as well for 2 micron targets with an earlier onset of the plateau for energies $\geq 3.2 \mathrm{MeV}$ at a divergence of $125 \mathrm{mrad}$. This is a direct consequence of the larger laser spot size compared to the accelerating distance, i.e., $w_{0} \gg \lambda_{D}$. The contribution to the divergence by scattering of the hot plasma electrons propagating through the solid target and their recirculation [37] is increased for thicker targets. As a result, the rear-side sheath field is transversally extended, leading to reduced proton beam divergence for thicker targets. Additionally, the dose deposited in the individual layers is almost twice as high for the 2 micron shot compared to the 5 micron shot with a total of $(7 \pm$ 2) $\times 10^{11}$ protons with energies $\geq 1.2 \mathrm{MeV}$.

Compared to other published experiments with $\mathrm{PW}$ lasers in the TNSA regime, the proton beam divergence was significantly reduced by a factor of 3-5 [36], reaching values of advanced acceleration schemes [38-40]. Together with the increased proton numbers, the charge density reached values so far only accessible with single shot laser systems and/or advanced acceleration mechanisms [3].

\section{PARTICLE-IN-CELL SIMULATION}

The relativistic fully electromagnetic particle-in-cell (PIC) simulation code, WARP [41,42], was used to model 

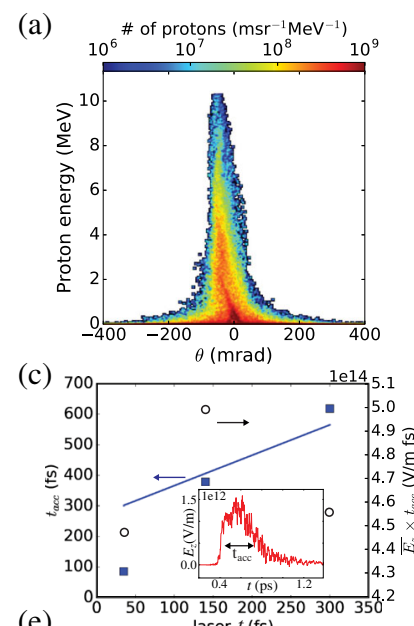

(e)

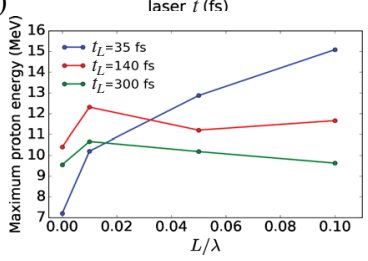

(b)

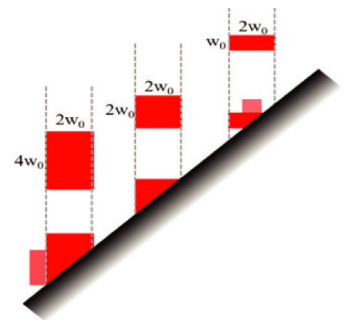

(d)

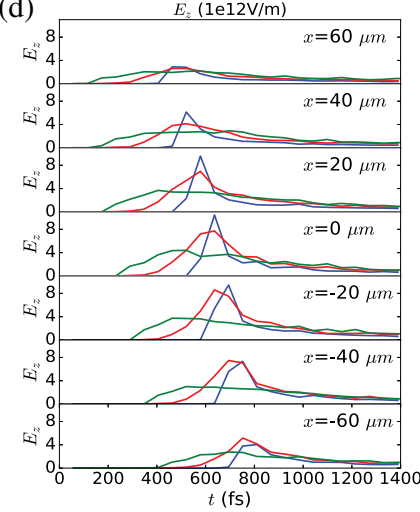

FIG. 4. (a) Simulated Energy vs angle distribution of protons in logarithmic color scales. (b) Sweeping effect shown schematically for three different laser pulse lengths as indicated in the figure. (c) Acceleration time $\left(t_{\mathrm{acc}}\right)$ defined as the $1 / e$ duration of $E_{z}$ acting on the protons with highest energy (see inset) as a function of a laser pulse duration (blue squares). Estimated interaction time $2 w_{0} / c+t_{L}$ (blue line). Impulse experience by a proton, electric field $\bar{E}_{z} \times t_{\text {acc }}$ (black circles). (d) $E_{z}$ as a function of simulation time at different transversal locations $x$ as indicated in the figure for laser pulse durations of $30 \mathrm{fs}$ (blue), $140 \mathrm{fs}$ (red) and $300 \mathrm{fs}$ (green). (e) Maximum proton energy for laser pulse durations of $30 \mathrm{fs}$ (blue), $140 \mathrm{fs}$ (red) and $300 \mathrm{fs}$ (green) as a function of $L$.

the experiment. The size of the simulation box was $\left(L_{z}, L_{x}\right)=(146 \mu \mathrm{m}, 357 \mu \mathrm{m})$ and the cell size was $0.0121 \lambda_{L} \times 0.0242 \lambda_{L}$, where $\lambda_{L}$ is the laser wavelength. The main target was composed of $\mathrm{Ti}^{11+}$ ions and electrons, initially located at $z=96 \mu \mathrm{m}$ with a thickness of $1 \mu \mathrm{m}$ along the $\mathrm{x}$ direction. The electron density of the target was $n_{e}=300 n_{\text {crit }}$, where $n_{\text {crit }}=\epsilon_{0} m_{e} \omega^{2} / e^{2}$ is the electron critical density, with $\epsilon_{0}$ the vacuum permittivity, $m_{e}, e$ electron mass and charge and $\omega$ the laser frequency.

To account for the effects of laser prepulses, we initialized preformed plasmas with exponential density profiles and scale lengths $L$ of $(0-0.05) \lambda_{L}$ and $0.01 \lambda_{L}$, at the front and back side, respectively, at a temperature equal to zero. To account for surface contamination, we added contaminant species, protons and $\mathrm{C}^{4+}$ ions on both sides of the target, with electron densities of $n_{e}=50 n_{\text {crit }}$ for the protons and $100 n_{\text {crit }}$ for the carbon ions. Both have exponential profiles with the same gradient scale length $L$. Convergence tests were used to determine numerical

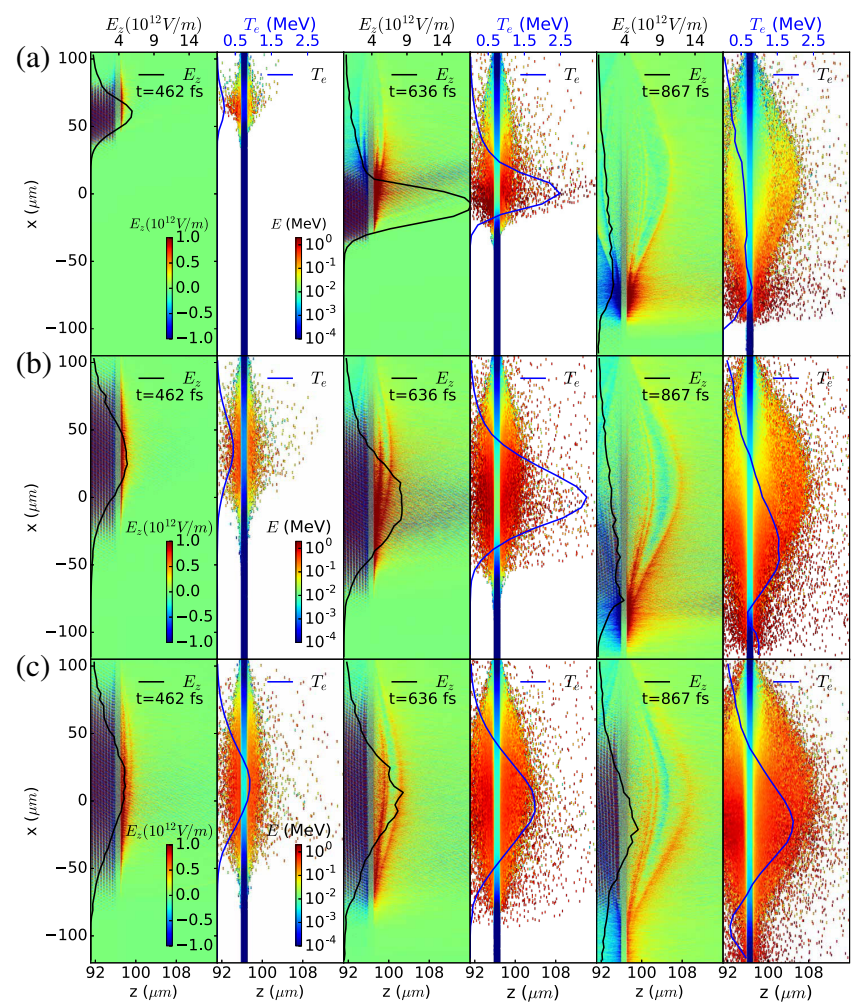

FIG. 5. (a) Evolution of longitudinal electric field $E_{z}$ and electron temperature $T_{e}$ at three different times during the interaction with $L=0.01 \lambda_{L}$, shown for three laser pulse duration in (a) $30 \mathrm{fs}$, (b) $140 \mathrm{fs}$ and (c) $300 \mathrm{fs}$. The time $t=636 \mathrm{fs}$ corresponds to the peak time of the spatiotemporal of the laser pulse at the front side of the target.

parameters, resulting in use of 120,40 , and 20 particles/ cell for Ti, carbon, and protons, respectively and electrons/ cell according to the ionization state given above. The center of the laser was initialized ahead of the target at $z=40 \mu \mathrm{m}$ as a Gaussian beam with the wavelength $\lambda_{L}=$ $0.8 \mu \mathrm{m}$ and was linearly polarized in the $\mathrm{x}-\mathrm{z}$ plane with peak intensities of $I=2.8-24 \times 10^{18} \mathrm{~W} / \mathrm{cm}^{2}\left(a_{0}=1.13\right.$, $1.65,3.3$ ), a focal spot of $w_{0}=50 \lambda_{L}$, and a pulse durations of $35,140,300 \mathrm{fs}$. It was obliquely incident on the target with the angle of 45 degrees. The target front side was initially located at $z=96 \mu \mathrm{m}$ and hence, the spatiotemporal peak of the laser arrived at the target at $t=614 \mathrm{fs}$. The simulation results are shown in Figs. 4 and 5.

Varying the initial preplasma scale length $L$ showed that a deviation from the linear intensity scaling of the maximum proton energy for $L \leq 0.02$ occurred as observed in the experiment [Fig. 4(e)]. Best agreement with the experimental results was obtained with $L=0.01 \lambda_{L}$ [Figs. 4(a) and 4(e)]. For these preplasma scale lengths, the dominant laser absorption mechanism is Brunel absorption [43]. The deviation from the linear intensity scaling is a result of the laser sweeping across the target front side if 
obliquely incident [Fig. 4(b)]. The temporal evolution of $E_{z}$ and $T_{e}$ (Fig. 5) demonstrates this sweeping effect. Where electron heating occurred very localized in case of the $30 \mathrm{fs}$ laser pulse, it was homogenized and most effective in the $140 \mathrm{fs}$ case that meets the condition $t_{L}=2 w_{0} / c$. Here, $E_{z}$ was almost constant along the transverse coordinate $x$ at the time of the maximum spatiotemporal laser intensity $(t=636 \mathrm{fs})$. This is also evident in the lineouts of the temporal evolution of the $E_{z}$ field at different transverse coordinates $x$ [Fig. 4(d)]. To quantify this effect, we defined the acceleration time $t_{\mathrm{acc}}$ as the $1 / e$ duration of $E_{z}$ [see inset Fig. 4(c)], experienced by the high energy protons and compared it to the laser target interaction time given by $2 w_{0} / c+t_{L}$ in Fig. 4(c). We found very good agreement for $t_{L} \geq 2 w_{0} / c$ and $t_{\text {acc }} \ll 2 w_{0} / c+t_{L}$ for $t_{L}<2 w_{0} / c$. The latter is due to transversely localized interaction of the short laser pulse. The impulse the proton experienced $\propto\left(\bar{E}_{z} \times\right.$ $\left.t_{\text {acc }}\right)$ is shown in Fig. 4(c) for $L=0.01 \lambda_{L}$. In case of preplasma gradients with $L \leq 0.02 \lambda_{L}$, the interaction time is the dominant factor, since laser absorption mainly occurs in plasma created by the main laser pulse. For longer preplasma scale lengths, the maximum $E_{z}$ field amplitude becomes more important, i.e., the initial preplasma conditions dominate the laser absorption process.

\section{CONCLUSIONS}

In conclusion, we have presented an experimental study of TNSA with large laser spot size, where an increased laser focal spot leads to sheath field geometries that accelerate ion beams of narrow and achromatic divergence at very high charge densities. In order to optimize the laser pulse duration, laser pulse sweeping across the target needed to be considered which leads to increased interaction times and optimum laser pulse duration of $2 w_{0} / c$. Such ion beams were generated at repetition rates of up to $0.5 \mathrm{~Hz}$, for the first time with lasers at petawatt peak power. It was shown that the accelerator performance allows tailoring of particle energy and numbers to the requirements of specific applications in the field of cancer cell biology, high energy density and material sciences. In particular, this could for the first time enable emittance preserving ion beam transport with a high-gradient active plasma lens to achieve a high ion peak fluence at nanosecond pulse durations.

\section{ACKNOWLEDGMENTS}

This work was supported by the U.S. Department of Energy Office of Science Offices of High Energy Physics and Fusion Energy Sciences, and by the Laboratory Directed Research and Development Program of Lawrence Berkeley National Laboratory under Contract No. DE-AC02-05CH11231. This research used resources (Edison, Cori) of NERSC, and an award of computation time by the INCITE program using resources of ALCF both supported by the Office of Science of the U.S. DOE under
Contracts No. DE-AC02-05CH11231 and No. AC0206CH11357, respectively. J. H. B. acknowledges financial support from the Alexander von Humboldt Foundation.

[1] H. Daido, M. Nishiuchi, and A. S. Pirozhkov, Review of laser-driven ion sources and their applications, Rep. Prog. Phys. 75, 056401 (2012).

[2] A. Macchi, M. Borghesi, and M. Passoni, Ion acceleration by superintense laser-plasma interaction, Rev. Mod. Phys. 85, 751 (2013).

[3] J. Schreiber, P. R. Bolton, and K. Parodi, Invited Review Article: "Hands-on" laser-driven ion acceleration: A primer for laser-driven source development and potential applications, Rev. Sci. Instrum. 87, 071101 (2016).

[4] S. V. Bulanov, J. J. Wilkens, T. Z. Esirkepov, G. Korn, G. Kraft, S. D. Kraft, M. Molls, and V. S. Khoroshkov, Laser ion acceleration for hadron therapy, Phys. Usp. 57, 1149 (2014).

[5] K. Krushelnick, E. L. Clark, R. Allott, F. N. Beg, C. N. Danson, A. Machacek, V. Malka, Z. Najmudin, D. Neely, P. A. Norreys et al., Ultrahigh-intensity laser-produced plasmas as a compact heavy ion injection source, IEEE Trans. Plasma Sci. 28, 1110 (2000).

[6] M. Borghesi, J. Fuchs, S. V. Bulanov, A. J. Mackinnon, P. K. Patel, and M. Roth, Fast ion generation by highintensity laser irradiation of solid targets and applications, Fusion Sci. Technol. 49, 412 (2006).

[7] M. Barberio, M. Sciscio, S. Vallieres, F. Cardelli, S. N. Chen, G. Famulari, T. Gangolf, G. Revet, A. Schiavi, M. Senzacqua et al., Laser-accelerated particle beams for stress testing of materials, Nat. Commun. 9, 372 (2018).

[8] R. L. Pease, A. H. Johnston, and J. L. Azarewicz, Radiation testing of semiconductor devices for space electronics, Proc. IEEE 76, 1510 (1988).

[9] B. Hidding, O. Karger, T. Konigstein, G. Pretzler, G. G. Manahan, P. McKenna, R. Gray, R. Wilson, S. M. Wiggins, G. H. Welsh et al., Laser-plasma-based space radiation reproduction in the laboratory, Sci. Rep. 7, 42354 (2017).

[10] M. Roth, T. E. Cowan, M. H. Key, S. P. Hatchett, C. Brown, W. Fountain, J. Johnson, D. M. Pennington, R. A. Snavely, S. C. Wilks et al., Fast Ignition by Intense Laser-Accelerated Proton Beams, Phys. Rev. Lett. 86, 436 (2001).

[11] V. Y. Bychenkov, W. Rozmus, A. Maksimchuk, D. Umstadter, and C. E. Capjack, Fast ignitor concept with light ions, Plasma Phys. Rep. 27, 1017 (2001).

[12] A. Macchi, A. Antonicci, S. Atzeni, D. Batani, F. Califano, F. Cornolti, J. J. Honrubia, T. V. Lisseikina, F. Pegoraro, and M. Temporal, Fundamental issues in fast ignition physics: from relativistic electron generation to proton driven ignition, Nucl. Fusion 43, 362 (2003).

[13] J. J. Honrubia, J. C. Fernndez, M. Temporal, B. M. Hegelich, and J. Meyer-ter-Vehn, Fast ignition of inertial fusion targets by laser-driven carbon beams, Phys. Plasmas 16, 102701 (2009).

[14] M. Roth, I. Alber, V. Bagnoud, C. Brown, R. Clarke, H. Daido, J. Fernandez, K. Flippo, S. Gaillard, C. Gauthier et al., Transport of laser accelerated proton beams and 
isochoric heating of matter, J. Phys. Conf. Ser. 244, 012009 (2010).

[15] P. K. Patel, A. J. Mackinnon, M. H. Key, T. E. Cowan, M. E. Foord, M. Allen, D. F. Price, H. Ruhl, P. T. Springer, and R. Stephens, Isochoric Heating of Solid-Density Matter with an Ultrafast Proton Beam, Phys. Rev. Lett. 91, 125004 (2003).

[16] C. Danson, D. Hillier, N. Hopps, and D. Neely, Petawatt class lasers worldwide, High Power Laser Sci. Eng. 3, e3 (2015).

[17] K. Nakamura, H. S. Mao, A. J. Gonsalves, H. Vincenti, D. E. Mittelberger, J. Daniels, A. Magana, C. Toth, and W. P. Leemans, Diagnostics, control and performance parameters for the BELLA high repetition rate petawatt class laser, IEEE J. Quantum Electron. 53, 1 (2017).

[18] K. W. D. Ledingham, P. R. Bolton, N. Shikazono, and C. M. C. Ma, Towards laser driven hadron cancer radiotherapy: A review of progress, Appl. Sci. 4, 402 (2014).

[19] R. A. Snavely, M. H. Key, S. P. Hatchett, T. E. Cowan, M. Roth, T. W. Phillips, M. A. Stoyer, E. A. Henry, T. C. Sangster, M. S. Singh et al., Intense High-Energy Proton Beams from Petawatt-Laser Irradiation of Solids, Phys. Rev. Lett. 85, 2945 (2000).

[20] S. C. Wilks, A. B. Langdon, T. E. Cowan, M. Roth, M. Singh, S. Hatchett, M. H. Key, D. Pennington, A. MacKinnon, and R. A. Snavely, Energetic proton generation in ultra-intense laser-solid interactions, Phys. Plasmas 8, 542 (2001).

[21] J. Fuchs, P. Antici, E. D'Humieres, E. Lefebvre, M. Borghesi, E. Brambrink, C. A. Cecchetti, M. Kaluza, V. Malka, M. Manclossi et al., Laser-driven proton scaling laws and new paths towards energy increase, Nat. Phys. 2, 48 (2006).

[22] F. Wagner, O. Deppert, C. Brabetz, P. Fiala, A. Kleinschmidt, P. Poth, V. A. Schanz, A. Tebartz, B. Zielbauer, M. Roth et al., Maximum Proton Energy above $85 \mathrm{MeV}$ from the Relativistic Interaction of Laser Pulses with Micrometer Thick $\mathrm{CH}_{2}$ Targets, Phys. Rev. Lett. 116, 205002 (2016).

[23] A. Higginson, R. J. Gray, M. King, R. J. Dance, S. D. R. Williamson, N. M. H. Butler, R. Wilson, R. Capdessus, C. Armstrong, J. S. Green et al., Near-100 MeV protons via a laser-driven transparency-enhanced hybrid acceleration scheme, Nat. Commun. 9, 724 (2018).

[24] D. Jung, L. Yin, D. C. Gautier, H.-C. Wu, S. Letzring, B. Dromey, R. Shah, S. Palaniyappan, T. Shimada, R. P. Johnson et al., Laser-driven $1 \mathrm{GeV}$ carbon ions from preheated diamond targets in the break-out afterburner regime, Phys. Plasmas 20, 083103 (2013).

[25] J. Fuchs, T. E. Cowan, P. Audebert, H. Ruhl, L. Gremillet, A. Kemp, M. Allen, A. Blazevic, J. C. Gauthier, M. Geissel et al., Spatial Uniformity of Laser-Accelerated UltrahighCurrent MeV Electron Propagation in Metals and Insulators, Phys. Rev. Lett. 91, 255002 (2003).

[26] J. S. Green, D. C. Carroll, C. Brenner, B. Dromey, P. S. Foster, S. Kar, Y. T. Li, K. Markey, P. McKenna, D. Neely et al., Enhanced proton flux in the $\mathrm{MeV}$ range by defocused laser irradiation, New J. Phys. 12, 085012 (2010).
[27] B. H. Shaw, S. Steinke, J. van Tilborg, and W. P. Leemans, Reflectance characterization of tape-based plasma mirrors, Phys. Plasmas 23, 063118 (2016).

[28] J. van Tilborg, S. Steinke, C. G. R. Geddes, N. H. Matlis, B. H. Shaw, A. J. Gonsalves, J. V. Huijts, K. Nakamura, J. Daniels, C. B. Schroeder et al., Active Plasma Lensing for Relativistic Laser-Plasma-Accelerated Electron Beams, Phys. Rev. Lett. 115, 184802 (2015).

[29] S. Ter-Avetisyan, M. Schnuerer, and P. V. Nickles, Time resolved corpuscular diagnostics of plasmas produced with high-intensity femtosecond laser pulses, J. Phys. D 38, 863 (2005).

[30] S. Steinke, J. van Tilborg, C. Benedetti, C. G. R. Geddes, C. B. Schroeder, J. Daniels, K. K. Swanson, A. J. Gonsalves, K. Nakamura, N. H. Matlis et al., Multistage coupling of independent laser-plasma accelerators, Nature (London) 530, 190 (2016).

[31] M. Noaman-ul-Haq, H. Ahmed, T. Sokollik, L. Yu, Z. Liu, X. Yuan, F. Yuan, M. Mirzaie, X. Ge, L. Chen et al., Statistical analysis of laser driven protons using a highrepetition-rate tape drive target system, Phys. Rev. Accel. Beams 20, 041301 (2017).

[32] P. V. Nickles, M. Schniirer, S. Steinke, T. Sokollik, S. Ter-Avetisyan, A. Andreev, and W. Sandner, Generation and manipulation of proton beams by ultra-short laser pulses, AIP Conf. Proc. 1153, 140 (2009).

[33] A. Flacco, F. Sylla, M. Veltcheva, M. Carri, R. Nuter, E. Lefebvre, D. Batani, and V. Malka, Dependence on pulse duration and foil thickness in high-contrast-laser proton acceleration, Phys. Rev. E 81, 036405 (2010).

[34] K. Zeil, S. D. Kraft, S. Bock, M. Bussmann, T. E. Cowan, T. Kluge, J. Metzkes, T. Richter, R. Sauerbrey, and U. Schramm, The scaling of proton energies in ultrashort pulse laser plasma acceleration, New J. Phys. 12, 045015 (2010).

[35] P. A. Seidl, J. J. Barnard, E. Feinberg, A. Friedman, E. P. Gilson, D. P. Grote, Q. Ji, I. D. Kaganovich, B. Ludewigt, A. Persaud et al., Irradiation of materials with short, intense ion pulses at NDCX-II, Laser Part. Beams 35, 373 (2017).

[36] F. Nuernberg, M. Schollmeier, E. Brambrink, A. Blazevic, D. C. Carroll, K. Flippo, D. C. Gautier, M. Geissel, K. Harres, B. M. Hegelich et al., Radiochromic film imaging spectroscopy of laser-accelerated proton beams, Rev. Sci. Instrum. 80, 033301 (2009).

[37] A. J. Mackinnon, Y. Sentoku, P. K. Patel, D. W. Price, S. Hatchett, M. H. Key, C. Andersen, R. Snavely, and R. R. Freeman, Enhancement of Proton Acceleration by Hot-Electron Recirculation in Thin Foils Irradiated by Ultraintense Laser Pulses, Phys. Rev. Lett. 88, 215006 (2002).

[38] S. Steinke, A. Henig, M. Schnuerer, T. Sokollik, P. V. Nickles, D. Jung, D. Kiefer, R. Hoerlein, J. Schreiber, T. Tajima et al., Efficient ion acceleration by collective laserdriven electron dynamics with ultra-thin foil targets, Laser Part. Beams 28, 215 (2010).

[39] J. H. Bin, W. J. Ma, K. Allinger, H. Y. Wang, D. Kiefer, S. Reinhardt, P. Hilz, K. Khrennikov, S. Karsch, X. Q. Yan et al., On the small divergence of laser-driven ion beams 
from nanometer thick foils, Phys. Plasmas 20, 073113 (2013).

[40] J. S. Green, N. P. Dover, M. Borghesi, C. M. Brenner, F. H. Cameron, D. C. Carroll, P. S. Foster, P. Gallegos, G. Gregori, P. McKenna et al., Enhanced proton beam collimation in the ultra-intense short pulse regime, Plasma Phys. Controlled Fusion 56, 084001 (2014).
[41] J. L. Vay, D. P. Grote, R. H. Cohen, and A. Friedman, Novel methods in the Particle-In-Cell accelerator CodeFramework Warp, Comput. Sci. Discovery 5, 014019 (2012).

[42] http://blast.lbl.gov/.

[43] F. Brunel, Not-So-Resonant, Resonant Absorption, Phys. Rev. Lett. 59, 52 (1987). 